101

\title{
A Group Decision Support System by Externalizing, Sharing and Reflecting Individual Viewpoints
}

\author{
Naotaka Kato, Masanobu Chujo and Susumu Kunifuji \\ Japan Advanced Institute of Science and Technology, HOKURIKU \\ 1-1 Asahidai, Tatsunokuchi, Ishikawa, 923-12, Japan \\ nkato@jaist.ac.jp, kuni@jaist.ac.jp
}

\begin{abstract}
This paper proposes a new type of group decision support system which focuses on facilitating processes of reaching a consensus. The system is characterized by the following points: (1) It is an interactive system which shows each decision maker in a group useful information for decision-making with GUI. (2) It extracts conflicts which should be adjusted among decision makers in the group by sharing their viewpoints. (3) It supports negotiation processes for resolving conflicts by using a tradeoff analysis based on sensitivity analysis. (4) It shows each decision maker the degree of agreements and the degree of compromises during the negotiation process. In this abstract, we describe the outline of our system and then show an implementation example. The experimental results shows some usefulness of the system for supporting negotiation processes by iterative interaction between each decision maker and the system.
\end{abstract}

\section{KEYWORDS GDSS, Groupware, tradeoff analysis, Analytic Hierarchy Process}

\section{INTRODUCTION}

Group decision support systems ( GDSSs ) (Gray, 1994) have a variety of methods for supporting consensus making processes. Generally, in these processes, each member has his individual viewpoint because he has his own standard of values. To reach a consensus effectively, each individual should externalize his viewpoint and then the members should reflect upon the viewpoints, and analyze them to reach a consensus. Our approach to decision-making based on reaching a consensus focuses on both disagreements and compromises that come from members' individual viewpoints, that is, which viewpoints lead to disagreements and which lead to compromises can be quantitatively represented.

In this abstract, we propose a novel interactive GDSS which brings to reality the above approach and includes two kinds of tradeoff analyses for resolving conflicts among the decision makers.

\section{PROPOSED GROUP DECISION SUPPORT METHOD}

Figure 1 shows a process of group decision-making based on alternative selection. It is composed of three steps. The first two steps are typical ones. The first step is for constructing an evaluation structure. All decision makers must have a common recognition of the decision-making problem. In this step, we use a well-known thinking support method for a group called the KJ method (Kawakita, 1975). It is used to analyze an unstructured decision-making problem and to construct a hierarchical evaluation structure for the problem.

Human-Computer Interaction: INTERACT'97 S. Howard, J. Hammond \& G. Lindgaard (editors)

Published by Chapman \& Hall OIFIP 1997 


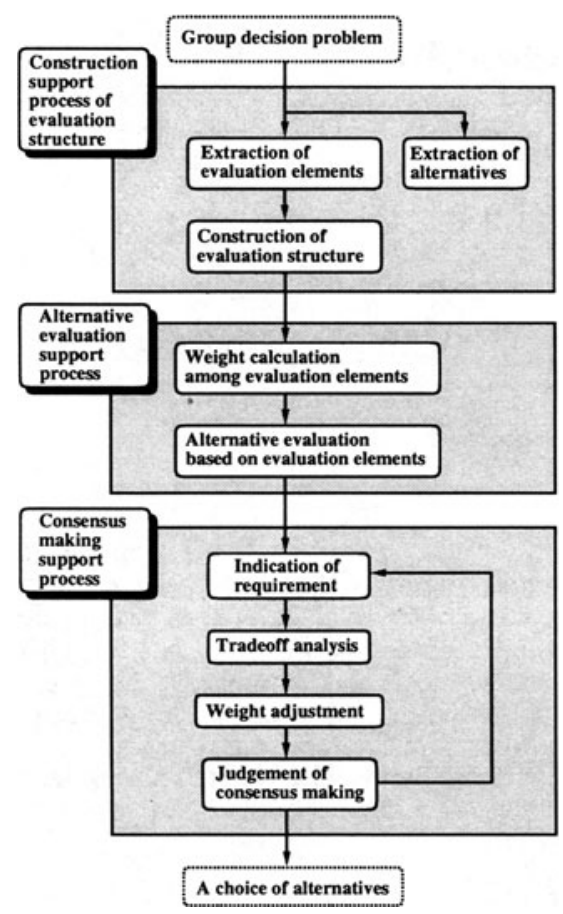

Figure 1 The proposed group decision support model

Second step is for evaluating alternatives based on the evaluation structure. AHP (Analytic Hierarchy Process) (Saaty, 1980) is used to evaluate alternatives quantitatively. It constructs an evaluation structure from individual's subjective judgements and then calculates the weights for each evaluation's attributes in the structure. A consistency ratio for member's judgements is also computed.

The last step is new proposed one for reaching a consensus among the members. Each individual viewpoint is represented as an evaluation structure with weights assigned to each attribute. Because members share their viewpoints, the viewpoint of one member may affect that of another. By analyzing the differences between viewpoints, we can find some conflicting parts of the viewpoints. Such conflicts are adjusted through negotiation processes based on tradeoff analysis. In the tradeoff analysis, we adapt the sensitivity analysis method (Masuda, 1987).

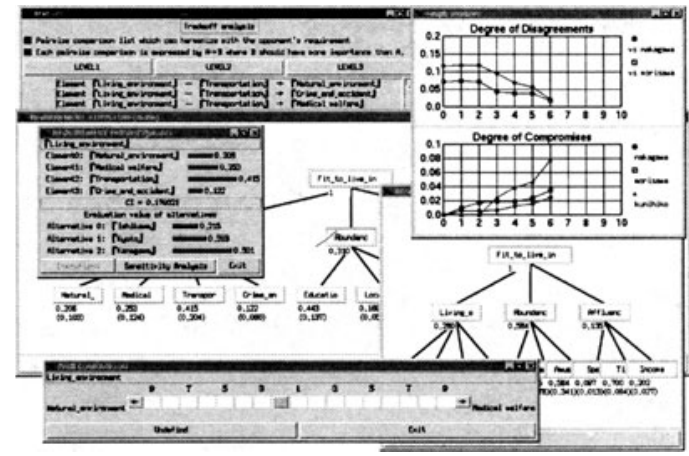

Figure 2 An example of windows for tradeoff analysis

\section{IMPLEMENTATION}

We have implemented a prototype system on a SUN work station with $\mathrm{X}$ window. The system has WYSIWIS (What You See Is What I See) function including GUI. Figure 2 shows a snapshot of operation windows in a process of the tradeoff analysis.

\section{CONCLUSION}

We observed the following usefulness of the system through some experimental evaluation: (1) The effect of individual variation in negotiation ability can be reduced in the negotiation processes. (2) Negotiation activity through this system becomes more persuasive or reasonable than conventional negotiation activity. (3) Decision makers become more amenable to compromising. (4) The system can also be used to enhance the effectiveness of the Delphi Technique.

\section{REFERENCES}

Gray, P (1994) Decision Support and Executive Information Systems, Prentice-Hall.

Kawakita, J. (1975) The KJ Method, Kawakita Research Institute.

Masuda, T. (1987) Sensitivity Coefficients of Consistency Index and Priorities Used in the Analytic Hierarchy Process, IEICE, A Vol.J70-A, No.11, 15621567 (in Japanese).

Saaty, T.L. (1980) The Analytic Hierarchy Process, McGraw-Hill. 